\title{
Exploring phase change materials in gloves to regulate body temperature
}

\author{
Shu-Hwa Lin / University of Hawaii at Manoa / Manoa / USA \\ Chih-Pong Chang / Chinese Culture Unversity / Taipei / Taiwan \\ Lynn M. Boorady / State University of New York College at Buffalo / Buffalo / USA \\ Susan P. Ashdown / Cornell University / Ithaca / USA
}

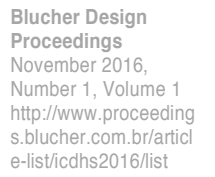

\begin{abstract}
There are two types of Phase Change Materials (PCMs) which have been developed and adopted in textiles: heat (energy released) and cool (energy absorbed). This paper discusses current PCM applications in gloves and explores future usage. Laboratory research design was used to explore the application of PCM in the design of protective gloves to determine if there was improved thermal regulation. Prototype gloves were developed using a 35\% cotton $/ 65 \%$ polyester blend interlock material which comprised of a top and bottom layer. The middle layer was a coated PCM fabric. A final layer using PANEX, a weave carbon fabric, surrounded the other layers and formed the outer shell of the glove. Preliminary tests indicate that absorbency or release of heat (energy) while changing states within this glove structure will provide a benefit for the wearer. Future research will test these gloves in working conditions.
\end{abstract}

\section{Keywords}

Personal Protective Equipment (PPE), gloves, Phase Change Materials (PCM), hand function

\section{Introduction}

Phase Change Materials (PCMs) have been suggested as latent energy storage materials because as they change phase from solid to liquid, liquid to gas and vice versa, energy in the form of heat is absorbed or released. This theory derives from the use of chemical bonds to store and release heat. The thermal energy transfer occurs when a material changes from a solid to a liquid, or from a liquid to a solid (Bendkowska, Tysiak, Grabowski, \& Blejzyk, 2005). This is called a change in state, or "phase." PCM, proven to possess thermal-regulating characteristics, is proposed for applications in clothing materials in conditions that require workers to face extreme temperatures. PCM also is believed to conserve energy and maintain certain temperatures, so PCMs have been chosen for latent energy storage materials.

The goal of PCM textiles is to create reusable energy to maintain body temperature, as well as to optimize the performance of protective wear such as gloves. When the wearer's body temperature increases or decreases, the PCMs applied to the fabric will change state helping to regulate the wearer's body temperature by providing warmth or cooling. Maintaining a stable hand temperature can improve working conditions and comfort. Hand function is compromised when hands become too cold, as dexterity and ability to manipulate materials is decreased.

In some work environments, jobs require the use of heavy garments or specially designed clothing with specific functions to protect the wearer from injury, heat stress, or contamination. These special-function garments could prevent transfer of hazardous materials onto the skin, as well as prevent the transfer of heat and moisture out of the garment. To accomplish this, researchers have developed a way to encapsulate PCMs into clothing fibers to reduce heat loss or prevent overheating in protective clothing (Mondal, 2008). PCMs react instantly to temperature changes. Because of the energy they release and absorb during these changes, PCMs can be used for thermal storage and thermal control.

Theoretically, PCM's ability to preserve energy is relatively simple. When a PCM's temperature increases above its melting point, the PCM absorbs and stores heat as thermal energy as it melts. When the PCM's temperature increases beyond the specified temperature range, the PCM is powered off and the PCM cools to below 
the melting point, releasing its stored energy and returning back to a solid state. As PCM absorbs heat, it provides thermal regulation to wearers, as well as enhances comfort by reducing perspiration. In this way, heat stress is prevented.

Another characteristic of PCM is that it acts as a mediator between the body and the environment's temperatures. PCM products will help people who need to protect their body during outdoor activities in severe conditions like extreme hot or cold (Flouris \& Cheung, 2006). In agriculture, industry, and recreation, many subjects may encounter severe sun exposure, which poses a risk to their health without proper protection against ultra-violet light. Taking such cases into consideration, another purpose of this project is to explore and enhance $\mathrm{PCM}$ product effectiveness relative to consumer needs.

The goal of PCM textiles is to create reusable energy to maintain body temperature, as well as to optimize the performance of protective wear such as gloves. Maintaining a stable hand temperature can improve working conditions and comfort. Hand function is compromised when they hand becomes too cold, as dexterity and ability to manipulate materials is decreased. The back of the hand also has blood vessels close to the surface and as blood runs through the veins the gloves will allow cooling to take place and help regulate temperature throughout the body. This project is intended to explore issues for the product development of PCMs to create gloves with improved thermal regulation properties. The study has practical applications for the inner linings of protective clothing in conditions of high heat or extreme temperature (Kovacs, Splittstoesser, Maronitis, \& Marras; 2002; Mondal, 2008). Laboratory research design was used in this study to explore the application of PCM in design protective gloves.

\section{Method}

\section{Procedure 1: Preparing the PCM}

The first step involved dissolving $3 \mathrm{~g}$ of three-isocyanate in $6 \mathrm{~g}$ of ethyl acetate in the homogenizer. Then $3 \mathrm{~g}$ of $\mathrm{n}$ eicosane was added and the solution was stirred until the n-eicosane was completely dissolved. Next, 10g of PVA aqueous solution ( $5 \%$ weight) was added to the solution. Once all chemicals were added, the solution was mixed in the homogenizer for ten minutes at a temperature of $40^{\circ} \mathrm{C}$ to create a completely homogeneous solution. Stirring was continued until polymerization occurred and the ethyl acetate was completely volatile.

In this study, a field emission scanning electron microscope was used to explore the hightenacity polyester fabric after the irradiation of argon and oxygen plasma treatment. Images were used to determine change in fiber surface according to the different variables: gas treatment, plasma power, and irradiation time. The operating current was $12 \mathrm{~mA}$ and the voltage was $10 \mathrm{kV}$. -3-5 contact angle and surface free energy.

Microencapsulated phase change materials (PCMs) were successfully prepared by three different process: emulsion non-solvent addition method, in-situ polymerization and interfacial polycondenation. The process parameters- such as polymerization temperature, additive, rotational speed and mass ration of phase change materials to monomer etc. - which control the size, shell/core ration and yield the microcapsule were studied. Chemical composition and microstructure of the microcapsules were characterized by FTIR and microscopy, and thermal characteristics were tested by differential scanning calorimetry. The results reveal that good microencapsulation can be achieved.

\section{Procedure 2: Designing gloves}

The following is the design and experiments design and analysis along with results. Two PCMs gloves were designed on different labs and tested.

The concern is that traditional leather gloves lose tactile sensation. It is easy to be tired and reduce productivity to expose this gap between the sleeve and the glove to a heat environment without any protection. Two prototypes were created and tested on male subjects to evaluate fit, mobiles, hand movements, and protective functions. This project is proposal to adopt PCMs to create fire fight's gloves to lower heat sensation, allow fire fighter's hands to be tactile sensitive, provide protection and make it easier for fire fighter's to use hands properly (grasp items, etc.). The design of the gloves can be slimmer due to the advance nature of the materials used which enhances mobility for the wearer. This usability testing is important to ensure that the fire fighters can use the gloves effectively.

The application of PCM in the design of protective gloves for firefighters was explored through laboratory research to determine if there was improved thermal regulation. An interlock material made of a 35\% cotton/65\% polyester blend was identified and used to develop prototype gloves. The interlock material had two 
layers and between these layers was a coated PCM fabric. All the layers were surrounded by PANEX, a woven carbon fabric, which formed an outer shell. This combination of layers would provide protection and cooling to the inter-lining section that covered hand back area exposed to surface.

Preliminary tests indicate that absorbency or release of heat (energy) while changing states within this gloves structure will provide a benefit for the firefighters. PCMs have been found to be one of the most efficient ways of storing thermal energy and it is expected that the use of this material in gloves will provide a new use for this developing material technology. Future research will test these gloves in working conditions.

Qualitative research design was used in this study. A self-developed questionnaire was used to interview subjects after they tried out the cooling gloves containing PCM. The questionnaire was revised after the pilot study. The questionnaire covered information about demographics with six questions, and explored the needs of PCM users in seven closed and two open-ended questions. The seven closed questions reflect consumer values, the effectiveness of PCM, and its availability in the market, while the open questions collect deeper insight into fire fighters' interests and the need for future product development.

\section{Discussion and Conclusion}

The results of this study suggest that consumers are interested in adopting PCM products because not only do PCMs contain an energy sustaining property, but they also provide adequate temperature control for the body. Suggestions for improvement were provided by each group of subjects. Among these subjects, color distinctions were suggested so the firefighter, rank and company could be better recognized in the field. Common concerns that were raised by each group included the lack of specific properties. Subjects agreed that the fire fighter traditional leather gloves provide great heat protection but loss in tactile properties reduce some capability and the gloves were not attached to their jacket creating coverage issues. Most subjects indicated that effectiveness of the leather gloves was limited by thickness of coverage on the hand and duration of use.

Many target consumers appreciated the immediate temperature changes of PCMs in other commercial products. Their absorbency or release of heat (energy) while changing states within a certain temperature range was considered beneficial by consumers. Products that include PCMs, such as gloves, have a high likelihood of being adopted by fire fighters. That apparel coated with PCM can either reduce heat loss or prevent overheating has already been accepted by some targeted consumer groups. Therefore, target consumers have embraced PCM as a thermal balance between heat generated by the body and heat released into the environment. $\mathrm{PCM}$ have been found to be one of the most efficient ways of storing thermal energy.

This study looked at the use of PCM textiles to regulate the temperature of the wearer, in this case fire fighter gloves. PCMs have been found to be one of the most efficient ways of storing thermal energy and it is expected that the use of this material in gloves will provide a new use for this developing material technology. In this study the new material was found to be a technical success and offers the additional benefit of less bulkiness leading to better mobility and tactile senses for the wearer. Future research will evaluate the gloves in working conditions.

\section{References}

Bendkowska, W., Tysiak, J., Grabowski, L., \& Blejzyk, A. (2005). Determining temperature regulating factor for apparel fabrics containing phase change material. International Journal of Clothing Science and Technology, 17 (3/4), 209-214.

Mondal, S. (2008). Phase change materials for smart textiles: An overview. Applied Thermal Engineering, 28 (11-12), 1536-1550.

Flouris, A., \& Cheung, S. (2006). Design and control optimization of microclimate liquid cooling systems underneath protective clothing. Annals of Biomedical Engineering, 34 (3), 359-372.

\section{Biographical note}

Dr. Chi-Pong Chang is currently serving in the Department of Textile Engineering, Chinese Culture University in Taiwan. His expertise is in textile chemistry, polymer chemistry and functional textiles. His research direction includes microcapsules containing phase change material, modified DNA applied on dyeing wastewater and fiber soilless cultivation. 
Dr. Shu-Hwa Lin is an Associate Professor in the Fashion Design \& Merchandising program at the University of Hawaii at Mānoa. Her research examines new forms of textiles and clothing that contribute to the health, protection, comfort, utility, and appearance concerns of individuals and groups with special needs.

Lynn M. Boorady, Ph.D. is an Associate Professor and Chair at SUNY - Buffalo State College in the Fashion and Textile Technology department. She is a Fulbright Scholar and invited speaker in her areas of expertise which include personal protective equipment, issues in sizing and fit and 3D body scanning.

Susan Ashdown is the Helen G. Canoyer Professor in the Department of Fiber Science \& Apparel Design at Cornell University. Her research addresses the impact of technology on design, 3D body scanning, apparel sizing and fit, functional apparel design, and interactions of materials and design. She teaches technical apparel design. 